\title{
COVID-19 and Kawasaki disease: an analysis using Google Trends
}

\author{
Mrinalini Dey ${ }^{1,2}$ (D) Sizheng Steven Zhao ${ }^{1,2}$ (D)
}

Received: 25 May 2020 / Revised: 25 May 2020 / Accepted: 9 June 2020 / Published online: 17 June 2020

(C) International League of Associations for Rheumatology (ILAR) 2020

COVID-19, a novel coronavirus disease, was declared a pandemic by the World Health Organisation on 11 March 2020. There have been 4,248,389 confirmed cases and 294,046 fatalities worldwide [1]. COVID-19 has been reported in children, although comprising $<2 \%$ of cases (https://www.rcpch. ac.uk/sites/default/files/generated-pdf/document/COVID19\%2D\%2D-research-evidence-summaries.pdf). Paediatric COVID-19 generally follows a milder course. However, there have been reports of a "Kawasaki-like" syndrome in children, first published in April 2020 [2]. This was followed by a case series in London, describing Kawasaki-like shock in children with COVID-19 [3], and reports of clusters in countries including Spain, Italy, and the USA [2].

Kawasaki disease is a medium-vessel vasculitis, usually affecting children $<5$ years. Features include fever, exanthema, lymphadenopathy, and mucosal changes. The most serious complication is coronary artery aneurysms, affecting up to $30 \%$ of patients [4]. The disease is more common in Southeast Asia, including Asian children living elsewhere, suggesting a genetic predisposition. Although the cause of Kawasaki disease is unknown, infection may play a role, especially given the seasonal prevalence of cases peaking during winter-spring [4].

Since early April, cases of hyperinflammatory shock in paediatric COVID-19 have continued to rise. We used Google Trends to analyse public searches on this association and Kawasaki disease itself, during February-May 2020. We previously reported on the utility of Google Trends to monitor popularity of search terms over time, particularly important in

Mrinalini Dey

mrinalini.dey@nhs.net

Sizheng Steven Zhao

S.Zhao8@liverpool.ac.uk

1 Institute of Life Course and Medical Sciences, University of Liverpool, William Henry Duncan Building, 6 West Derby Street, Liverpool L7 8TX, UK

2 Rheumatology, Aintree Hospital, Liverpool University Hospitals NHS Foundation Trust, Lower Lane, Liverpool L9 7AL, UK rapidly progressive outbreaks of novel infections [5]. Accounting for $75 \%$ of internet searches worldwide, Google Trends is a useful platform to observe global and regional variations in interest in a given topic.

We conducted a Google Trends search on 14 May 2020 to first compare internet searches for "Kawasaki disease" during February-May 2020, to the same period in 2019. Figure 1 demonstrates similar public interest in Kawasaki disease in 2019 and 2020, until April of this year, when there is a sharp rise following the first reports of Kawasaki-like disease in childhood cases of COVID-19. Of note, searches related to Kawasaki disease were most popular in Indonesia, Philippines, and Malaysia during February-May 2019 (population-adjusted), as per the usual epidemiology of this disease. However, in the same period in 2020, the search term was most popular in France, Switzerland, and Italy, possibly reflecting the novel paediatric manifestation of COVID-19 emerging in these populations.

Google Trends data for searches incorporating both "Kawasaki" and "COVID-19" (or similar) are plotted alongside worldwide cumulative cases of COVID-19 since February 2020 in Fig. 1b. Search trends prior to this showed minimal activity and were therefore not included. Our analysis revealed a sharp increase in searches for "Kawasaki" and COVID-19-related terms combined, first in mid-April, then in May (although, this increase has not peaked as of yet). During April-May, this search was most popular overall in USA, Canada, France, and Italy. Smaller isolated peaks in searches for "Kawasaki COVID-19" are noted increasingly throughout February-April. This possibly reflects earlier unreported cases of hyperinflammatory Kawasaki-like syndrome associated with COVID-19. Our Google Trend analyses are limited by lack of information from other search engines, and lack of user demographics. It also does not accurately represent searches performed in areas with limited internet access.

In conclusion, Google Trends analyses indicate continued global interest in the possible association between COVID-19 and Kawasaki disease. This association may have been noted in paediatric populations prior to the published reports in early 
Popularity of searches for "Kawasaki disease" February-May 2019 \& 2020

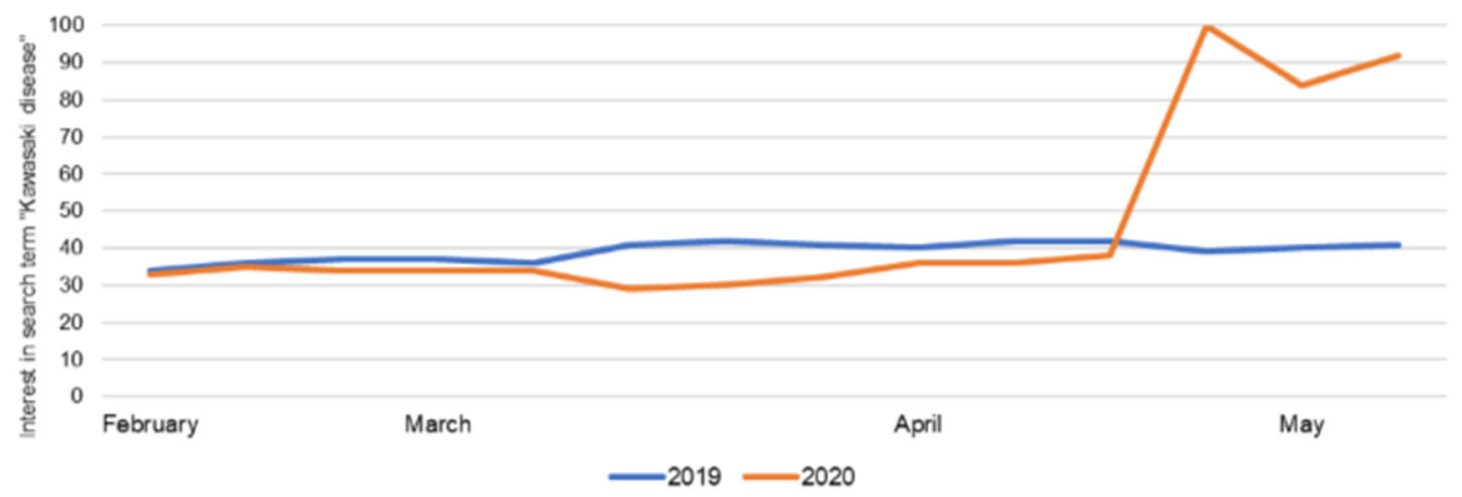

b Popularity of searches for combined "Kawasaki" and "COVID-19"-related terms

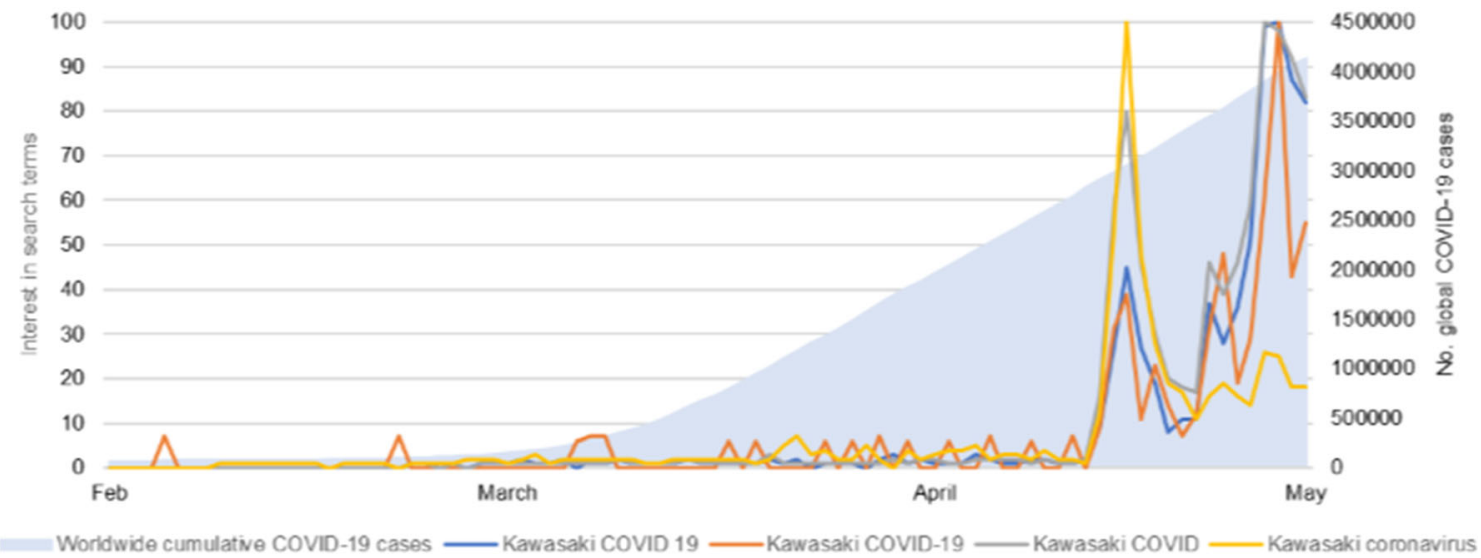

Fig. 1 a Popularity in Google searches for "Kawasaki disease", February-May 2019 and 2020. Values are relative to highest peak; value of 100 denotes peak popularity for the term. A score of 0 indicates insufficient data for the term. b Popularity in Google searches for terms containing "Kawasaki” plus "COVID-19" (or similar). Left axis denoted interest in search terms. Right axis denotes number of confirmed global cases of COVID-19 over the same period
April, leading to small increases in searches during FebruaryApril. Google Trends may prove useful in regional monitoring of the COVID-19 pandemic, especially given its ability to stratify results by country. It can therefore potentially be used to crudely monitor clinical features, including possible Kawasaki disease, and identify disease clusters in real-time.

\section{Compliance with ethical standards}

\section{Disclosures None.}

\section{References}

1. Coronavirus disease 2019 [Internet]. [cited 2020 May 14]. Available from: https://www.who.int/emergencies/diseases/novel-coronavirus2019
2. Jones VG, Mills M, Suarez D, Hogan CA, Yeh D, Bradley Segal J et al (2020) COVID-19 and Kawasaki disease: novel virus and novel case. Hosp Pediatr 7

3. Riphagen S, Gomez X, Gonzalez-Martinez C, Wilkinson N, Theocharis P. Hyperinflammatory shock in children during COVID-19 pandemic. Lancet (London, England) [Internet]. 2020 [cited 2020 may 14];0(0). Available from: http://www.ncbi.nlm. nih.gov/pubmed/32386565

4. Soni PR, Noval Rivas M, Arditi M. A comprehensive update on Kawasaki disease vasculitis and myocarditis. Vol. 22, Current Rheumatology Reports. Springer; 2020. p. 1-11

5. Dey M, Zhao SS, Goodson N. Global public interest in infectious and non-infectious arthritis: an evaluation using Google Trends. Rheumatology [Internet]. 2019 Jul 13; Available from: https:/doi. org/10.1093/rheumatology/kez283

Publisher's note Springer Nature remains neutral with regard to jurisdictional claims in published maps and institutional affiliations. 